\title{
Cold-fluid theory of equilibrium and stability of a high-intensity periodically twisted ellipse-shaped charged-particle beam
}

\author{
Jing Zhou, Ronak Bhatt, and Chiping Chen \\ Plasma Science and Fusion Center, Massachusetts Institute of Technology, Cambridge, Massachusetts 02139, USA
}

(Received 14 January 2005; published 3 March 2006)

\begin{abstract}
It is shown that there exists an exact paraxial cold-fluid equilibrium of a high-intensity, space-chargedominated charged-particle beam with a periodically twisted elliptic cross section in a nonaxisymmetric periodic magnetic field. Generalized envelope equations, which determine the beam envelopes, ellipse orientation, density, and internal flow velocity profiles, are derived. Nonrelativistic and relativistic examples of such beam equilibria are presented. The equilibrium and stability of such beams are demonstrated by self-consistent particle-in-cell (PIC) simulations.
\end{abstract}

DOI: 10.1103/PhysRevSTAB.9.034401

PACS numbers: 41.75.- $\mathrm{i}$, 41.85.Ja, 41.85.Lc, 52.59.Sa

\section{INTRODUCTION}

High-intensity ribbon (thin sheet) beams are of great interest for applications in particle accelerators, such as storage rings and $\mathrm{rf}$ and induction linacs, as well as vacuum electron devices, such as klystrons and traveling-wave tubes with periodic permanent magnet (PPM) focusing. They have the following remarkable properties. First, they can transport large amounts of beam currents at reduced intrinsic space-charge forces and energies. Second, they couple efficiently to rectangular rf structures. The combination of the space-charge reduction and efficient coupling allows efficient rf generation in vacuum electronic devices, and efficient acceleration in particle accelerators. Third, elliptic beams provide an additional adjustable parameter (e.g., the aspect ratio) which may be useful for better matching a beam into a periodic focusing channel.

Although ribbon beams have been discussed in the literature for four decades, the equilibrium of a highintensity, space-charge-dominated beam with a large-aspect-ratio elliptic cross section has not been discovered until this paper. Sturrock [1] first suggested the use of a periodic magnetic focusing consisting of an array of planar-wiggler magnets for rectilinear beams. Zhang et al. [2] had some modest success in the experimental demonstration of the transport of a low-intensity (10 A, $500 \mathrm{kV}$ ) sheet beam in a planar-wiggler magnetic field, and observed considerable beam loss. Researchers made use of the multiple-time-scale analysis and the paraxial approximations to obtain the smooth-beam approximation of high-intensity ribbon-beam equilibria [3-6]. In the smooth-beam approximation, the effects of the planarwiggler field [1,2], or the periodic cusp magnetic (PCM) field $[3,4]$, or the hybrid of PCM and periodic quadrupole magnet (PQM) fields [5,6], or the offset pole PCM field [6] are treated as some averaged focusing constants in the transverse directions. While the smooth-beam approximation yields simple results, the multiple-time-scale analysis requires time averaging, losing the detailed information about the beam dynamics. As a result, it does not provide adequate information about how to generate high-intensity ribbon beams in practice.

A widely used tool for the determination of evolution of the charged-particle beam systems is based on the rms beam description. The construction of self-consistent beam distributions with linear focusing forces is discussed in Refs. [7,8], such as Kapchinskij-Vladmirskij equilibrium [7-10] in an alternating-gradient quadrupole magnetic focusing field and the periodically focused rigid-rotor Vlasov equilibrium [11,12] in a periodic solenoid magnetic focusing field. For ultrahigh-brightness beams with negligibly small emittance, a cold-fluid theory can be used to examine the detailed information about the beam dynamics, especially the evolution of the density and flow velocity profiles [13-15], such as the cold-fluid corkscrewing equilibrium $[13,14]$ in a linear focusing channel consisting of solenoidal and quadrupole magnetic focusing fields.

In this paper, we present the cold-fluid equilibrium theory of an ultrahigh-brightness, space-charge-dominated, large-aspect-ratio beam propagating through a nonaxisymmetric periodic magnetic focusing field. Our beam distribution satisfies the assumptions for the distribution functions described generally in Refs. [7,8]. A paraxial cold-fluid model is employed to derive generalized envelope equations which determine the equilibrium flow properties of ellipse-shaped beams with negligibly small emittance. A matched envelope solution is obtained numerically from the generalized envelope equations, and the results show that the beam edges in both transverse directions are well confined, and that the angle of the beam ellipse exhibits a periodic small-amplitude twist. Twodimensional (2D) particle-in-cell (PIC) simulations with our Periodic Focused Beam 2D (PFB2D) code show good agreement with the predictions of equilibrium theory as well as beam stability.

The organization of the present paper is as follows. In Sec. II, the cold-fluid equilibrium theory of a periodically twisted ellipse-shaped charged-particle beam is presented 
and the generalized envelope equations are derived. In Sec. III, the generalized envelope equations are solved numerically for several examples of periodically twisted ellipse-shaped charged-particle beams. In Sec. IV, PIC simulations are used to verify the theoretical results. Conclusions are presented in Sec. V.

\section{COLD-FLUID EQUILIBRIUM THEORY}

We consider a high-intensity, space-charge-dominated beam, in which kinetic (emittance) effects are negligibly small. The beam can be adequately described by cold-fluid equations. In the paraxial approximation, the steady-state cold-fluid equations for time-stationary flow $(\partial / \partial t=0)$ in cgs units are $[13,14]$

$$
\begin{aligned}
& \beta_{b} c \frac{\partial}{\partial s} n_{b}+\nabla_{\perp} \cdot\left(n_{b} \mathbf{V}_{\perp}\right)=0 \\
& \nabla_{\perp}^{2} \phi^{s}=\beta_{b}^{-1} \nabla_{\perp}^{2} A_{z}^{s}=-4 \pi q n_{b}, \\
& n_{b}\left(\beta_{b} c \frac{\partial}{\partial s}+\mathbf{V}_{\perp} \cdot \nabla_{\perp}\right) \mathbf{V}_{\perp}=\frac{q n_{b}}{\gamma_{b} m}\left[-\frac{1}{\gamma_{b}^{2}} \nabla_{\perp} \phi^{s}+\beta_{b} \hat{\mathbf{e}}_{z}\right. \\
& \left.\times \mathbf{B}_{\perp}^{\mathrm{ext}}+\frac{\mathbf{V}_{\perp}}{c} \times B_{z}^{\mathrm{ext}}(s) \hat{\mathbf{e}}_{z}\right]
\end{aligned}
$$

where $s=z, \mathbf{x}_{\perp}=x \hat{\mathbf{e}}_{x}+y \hat{\mathbf{e}}_{y}, \nabla_{\perp}=\partial / \partial \mathbf{x}_{\perp}, q$ and $m$ are the particle charge and rest mass, respectively, $n_{b}$ is the particle density, $\mathbf{V}_{\perp}$ is the transverse flow velocity, $\gamma_{b}=$ $\left(1-\beta_{b}^{2}\right)^{-1 / 2}$ is the relativistic mass factor, use has been made of $\beta_{z}=V_{z} / c \cong \beta_{b}=$ const, $c$ is the speed of light in vacuum and the self-electric field $\mathbf{E}^{s}$ and self-magnetic field $\mathbf{B}^{s}$ are determined from the scalar potential $\phi^{s}$ and vector potential $A_{z}^{s} \hat{\mathbf{e}}_{z}$, i.e., $\mathbf{E}^{s}=-\nabla_{\perp} \phi^{s}$ and $\mathbf{B}^{s}=\nabla_{\perp} \times$ $A_{z}^{s} \hat{\mathbf{e}}_{z}$.

For the beam dimensions small relative to the characteristic scale of magnetic variations, i.e., $\left(k_{0 x} x\right)^{2} / 6 \ll 1$ and $\left(k_{0 y} y\right)^{2} / 6 \ll 1$, a three-dimensional (3D) nonaxisymmetric periodic magnetic field can be described to the lowest order in the transverse dimension as

$$
\begin{aligned}
\mathbf{B}^{\mathrm{ext}}(\mathbf{x}) \approx & B_{0}\left[\frac{k_{0 x}^{2}}{k_{0}} \cos \left(k_{0} s\right) x \hat{\mathbf{e}}_{x}+\frac{k_{0 y}^{2}}{k_{0}} \cos \left(k_{0} s\right) y \hat{\mathbf{e}}_{y}\right. \\
& \left.-\sin \left(k_{0} s\right) \hat{\mathbf{e}}_{z}\right]
\end{aligned}
$$

where $k_{0}=2 \pi / S, k_{0 x}^{2}+k_{0 y}^{2}=k_{0}^{2}$, and $S$ is the axial periodicity length. The 3D magnetic field in Eq. (4) is fully specified by the three parameters $B_{0}, S$, and $k_{0 x} / k_{0 y}$.

We seek solutions to Eqs. (1)-(3) of the form

$$
n_{b}\left(\mathbf{x}_{\perp}, s\right)=\frac{N_{b}}{\pi a(s) b(s)} \Theta\left[1-\frac{\tilde{x}^{2}}{a^{2}(s)}-\frac{\tilde{y}^{2}}{b^{2}(s)}\right]
$$

$$
\begin{aligned}
\mathbf{V}_{\perp}\left(\mathbf{x}_{\perp}, s\right)= & {\left[\mu_{x}(s) \tilde{x}-\alpha_{x}(s) \tilde{y}\right] \beta_{b} c \hat{\mathbf{e}}_{\tilde{x}} } \\
& +\left[\mu_{y}(s) \tilde{y}+\alpha_{y}(s) \tilde{x}\right] \beta_{b} c \hat{\mathbf{e}}_{\tilde{y}} .
\end{aligned}
$$

In Eqs. (5) and (6), $\mathbf{x}_{\perp}=\tilde{x} \hat{\mathbf{e}}_{\tilde{x}}+\tilde{y} \hat{\mathbf{e}}_{\tilde{y}}$ is a transverse displacement in the twisted coordinate system illustrated in Fig. 1; $\theta(s)$ is the twist angle of the ellipse; $\Theta(x)=1$ if $x>0$ and $\Theta(x)=0$ if $x<0$; and the functions $a(s), b(s)$, $\mu_{x}(s), \mu_{y}(s), \alpha_{x}(s), \alpha_{y}(s)$, and $\theta(s)$ are to be determined self-consistently [see Eqs. (8)-(12)].

The self-electric and self-magnetic fields are well known for an elliptical beam with density distribution specified in Eq. (5), i.e.,

$$
\phi^{s}=\beta_{b}^{-1} A_{z}^{s}=-\frac{2 q N_{b}}{a+b}\left(\frac{\tilde{x}^{2}}{a}+\frac{\tilde{y}^{2}}{b}\right) .
$$

Using the expressions in Eqs. (4)-(7), it can be shown that both the equilibrium continuity equation (1) and force equation (3) are satisfied if the dynamical variables $a(s)$, $b(s), \mu_{x}(s) \equiv a^{-1} d a / d s, \mu_{y}(s) \equiv b^{-1} d b / d s, \alpha_{x}(s), \alpha_{y}(s)$ and $\theta(s)$ obey the generalized beam envelope equations (see appendix)

$$
\begin{aligned}
& \frac{d^{2} a}{d s^{2}}-\left[\frac{b^{2}\left(\alpha_{x}^{2}-2 \alpha_{x} \alpha_{y}\right)+a^{2} \alpha_{y}^{2}}{a^{2}-b^{2}}\right. \\
& +\sqrt{\kappa_{z 0}} \frac{k_{0 x}^{2}-k_{0 y}^{2}}{k_{0}} \cos \left(k_{0} s\right) \sin (2 \theta) \\
& \left.\quad-2 \sqrt{\kappa_{z 0}} \alpha_{y} \sin \left(k_{0} s\right)\right] a-\frac{2 K}{a+b}=0,
\end{aligned}
$$

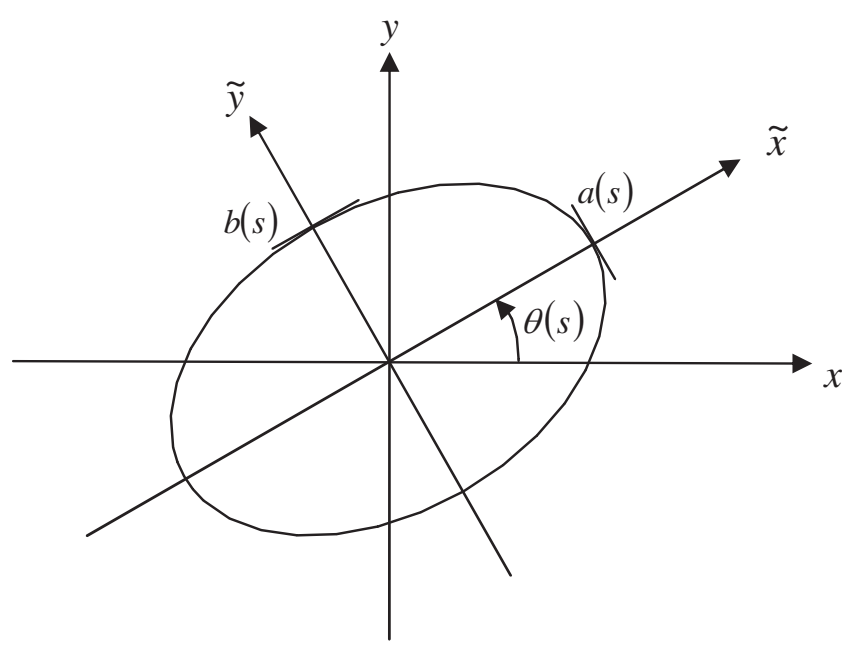

FIG. 1. Laboratory and twisted coordinate systems. 


$$
\begin{gathered}
\frac{d^{2} b}{d s^{2}}+\left[\frac{a^{2}\left(\alpha_{y}^{2}-2 \alpha_{x} \alpha_{y}\right)+b^{2} \alpha_{x}^{2}}{a^{2}-b^{2}}\right. \\
+\sqrt{\kappa_{z 0}} \frac{k_{0 x}^{2}-k_{0 y}^{2}}{k_{0}} \cos \left(k_{0} s\right) \sin (2 \theta) \\
\left.\quad+2 \sqrt{\kappa_{z 0}} \alpha_{x} \sin \left(k_{0} s\right)\right] b-\frac{2 K}{a+b}=0, \\
\frac{d}{d s}\left(a^{2} \alpha_{y}\right)-\frac{a b^{3}\left(\alpha_{x}-\alpha_{y}\right)}{a^{2}-b^{2}} \frac{d}{d s}\left(\frac{a}{b}\right) \\
-2 \sqrt{\kappa_{z 0}} \cos \left(k_{0} s\right) \frac{k_{0 x}^{2} \cos ^{2} \theta+k_{0 y}^{2} \sin ^{2} \theta}{k_{0}} a^{2} \\
-2 \sqrt{\kappa_{z 0}} a \frac{d a}{d s} \sin \left(k_{0} s\right)=0, \\
\frac{d}{d s}\left(b^{2} \alpha_{x}\right)-\frac{a^{3} b\left(\alpha_{x}-\alpha_{y}\right)}{a^{2}-b^{2}} \frac{d}{d s}\left(\frac{b}{a}\right) \\
-2 \sqrt{\kappa_{z 0}} \cos \left(k_{0} s\right) \frac{k_{0 x}^{2} \sin ^{2} \theta+k_{0 y}^{2} \cos ^{2} \theta}{k_{0}} b^{2} \\
-2 \sqrt{\kappa_{z 0}} b \frac{d b}{d s} \sin \left(k_{0} s\right)=0, \\
\frac{d \theta}{d s}=\frac{a^{2} \alpha_{y}-b^{2} \alpha_{x}}{a^{2}-b^{2}},
\end{gathered}
$$

where

$$
\sqrt{\kappa_{z 0}} \equiv \frac{q B_{0}}{2 \gamma_{b} \beta_{b} m c^{2}} \quad \text { and } \quad K \equiv \frac{2 q^{2} N_{b}}{\gamma_{b}^{3} \beta_{b}^{3} m c^{2}}
$$

Equations (8)-(12) have "time" reversal symmetry under the transformation $\left(s, a, b, a^{\prime}, b^{\prime}, \alpha_{x}, \alpha_{y}, \theta\right) \rightarrow$ $\left(-s, a, b,-a^{\prime},-b^{\prime},-\alpha_{x},-\alpha_{y}, \theta\right)$. This implies that the dynamical system described by Eqs. (8)-(12) has the hypersymmetry plane $\left(a^{\prime}, b^{\prime}, \alpha_{x}, \alpha_{y}\right)$.

\section{EXAMPLES OF PERIODICALLY TWISTED ELLIPSE-SHAPED CHARGED-PARTICLE BEAMS}

A numerical module in the PFB2D code has been developed to solve the generalized envelope equations (8)-(12). There are, in total, seven functions $a(s), b(s), a^{\prime}(s), b^{\prime}(s)$, $\alpha_{x}(s), \alpha_{x}(s)$, and $\theta(s)$ to be determined. The time reversal symmetry of the dynamical system requires the quantities $\left(a^{\prime}, b^{\prime}, \alpha_{x}, \alpha_{y}\right)$ vanish at $s=0$ for matched solutions, therefore, only the three initial values $a(0), b(0)$, and $\theta(0)$ corresponding to a matched solution need to be determined with Newton's method.

As an example, we consider a relativistic elliptic beam with $V_{b}=198.5 \mathrm{keV}$, current $I_{b}=85.5 \mathrm{~A}$, aspect ratio $a / b=5$, and nonaxisymmetric periodic permanent magnet focusing with $B_{0}=2.4 \mathrm{kG}, \quad S=2.2 \mathrm{~cm}$, and $k_{0 y} / k_{0 x}=1.52$ (see Table I, Col. 4). [We propose to use it in a $10 \mathrm{MW}$ L-Band ribbon-beam klystron for the International Linear Collider.] For such a system the matched solution of the generalized envelope equations (8)-(12) is calculated numerically as shown in Figs. 2(a)-2(c) (solid curves) with the corresponding parameters: $k_{0 x}=1.57 \mathrm{~cm}^{-1}, \quad k_{0 y}=2.39 \mathrm{~cm}^{-1}, \quad \sqrt{\kappa_{z 0}}=$ $0.732 \mathrm{~cm}^{-1}$, and $K=1.13 \times 10^{-2}$. The solution to the generalized envelope equations (8)-(12), displayed as solid and dashed curves in Fig. 2, shows that the semiaxes of the elliptical beam remain roughly constant with small oscillations, that the orientation of the ellipse twists periodically with an amplitude of 8.8 degrees, and that the normalized rotation flow velocities $\alpha_{x}$ and $\alpha_{y}$ oscillate with the magnet periodicity. It is worthwhile pointing out that the normalized velocities $\mu_{x}, \mu_{y}, \alpha_{x}$, and $\alpha_{y}$ vanish at $s=0$ which makes it a natural matching point for a parallel-flow beam with negligibly small emittance [16].

These high-intensity twisted elliptic-beam equilibria exist over a wide region of parameters ranging from the nonrelativistic to relativistic regimes. In addition to the relativistic elliptic-beam equilibrium discussed above, we present a nonrelativistic elliptic-beam equilibrium and a mildly relativistic elliptic-beam equilibrium in Table I. The

TABLE I. System parameters for elliptic-beam examples.

\begin{tabular}{lccc}
\hline \hline $\begin{array}{l}\text { Parameter } \\
\text { Application }\end{array}$ & $\begin{array}{c}\text { Nonrelativistic } \\
\text { Wireless communication }\end{array}$ & $\begin{array}{c}\text { Mildly relativistic } \\
\text { Missile defense }\end{array}$ & $\begin{array}{c}\text { Fully relativistic } \\
\text { International Linear Collider }\end{array}$ \\
\hline Frequency $(\mathrm{GHz})$ & 1.95 & 2.8 & 1.3 \\
rf Power $(\mathrm{kW})$ & $0.2(\mathrm{cw})$ & $500(\mathrm{pulsed})$ & $10000(\mathrm{pulsed})$ \\
Current $(\mathrm{A})$ & 0.11 & 18.5 & 85.5 \\
Voltage $(\mathrm{kV})$ & 2.29 & 45.0 & 198.5 \\
$\mathrm{~S}(\mathrm{~cm})$ & 1.912 & 2.626 & 2.2 \\
$k_{0 x} / k_{0 y}$ & 1.60 & 1.44 & 1.52 \\
$B_{0}(\mathrm{kG})$ & 0.337 & 1.099 & 2.40 \\
$a / b$ & 6.0 & 4.0 & 5.0 \\
$a(\mathrm{~cm})$ & 0.373 & 0.585 & 0.425 \\
$\theta_{\max }(\mathrm{deg})$ & 10.4 & 11.5 & 8.8 \\
\hline \hline
\end{tabular}


nonrelativistic elliptic beam corresponds to a beam design for a high-efficiency $200 \mathrm{~W}$ ribbon-beam amplifier (RBA) which is being developed at Massachusetts Institute of Technology (MIT) for communication, whereas the mildly
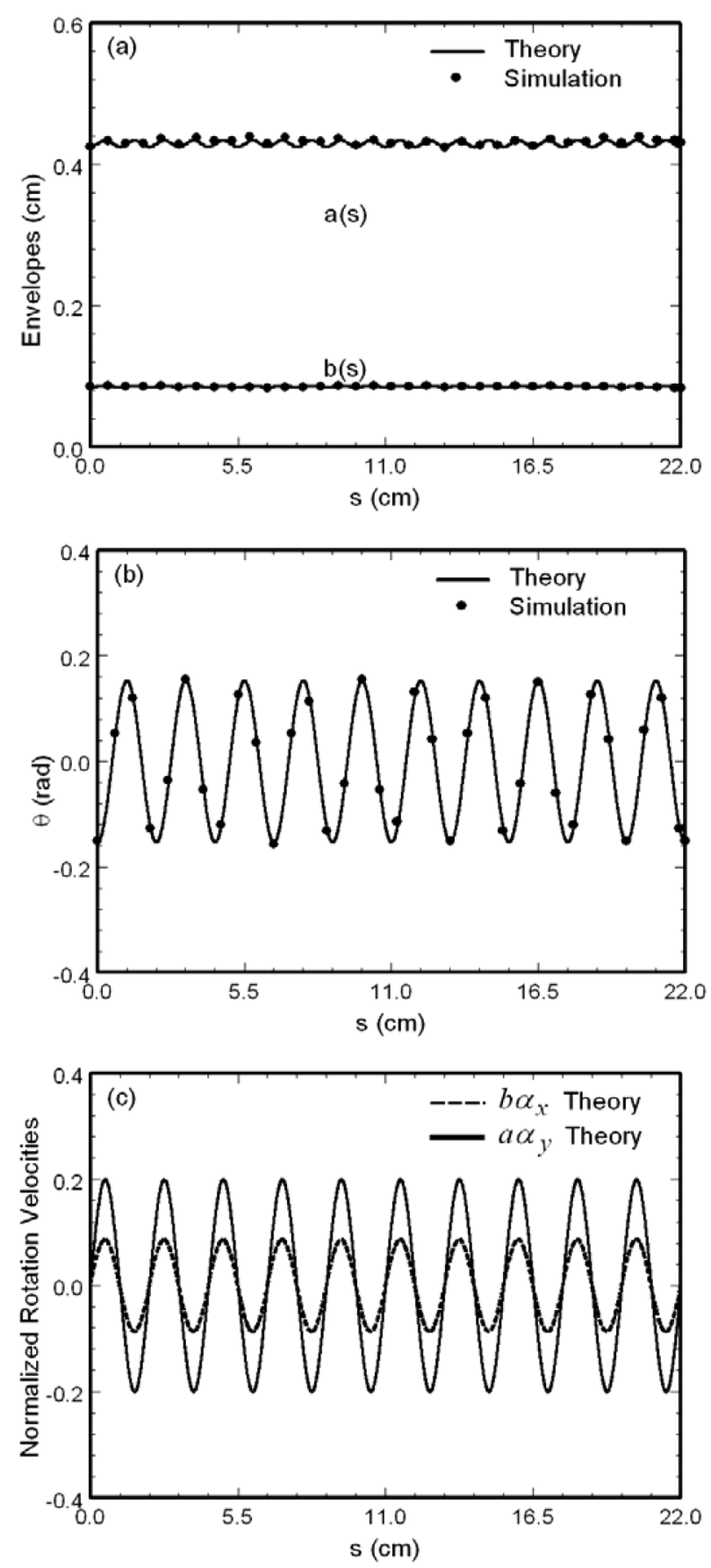

FIG. 2. Plots of (a) envelopes $a(s)$ and $b(s)$, (b) twist angle $\theta(s)$, and (c) normalized rotational velocities $b(s) \alpha_{x}(s)$ and $a(s) \alpha_{y}(s)$ versus the axial distance $s$ for the fully relativistic twisted ellipse-shaped beam in Table I (column 4). The solid and dashed curves are the generalized envelope solution, whereas the dotted curves are from the PFB2D simulation. relativistic elliptic beam is proposed for a high-power, high-efficiency RBA for radar applications.

\section{PIC SIMULATIONS}

The beam equilibria predicted by the generalized envelope equations are verified by 2D PIC simulations using the PFB2D code. In the PFB2D simulations, we use the paraxial field in Eq. (4), typically $5 \times 10^{5}$ particles, a square grid with $400 \times 400$ cells, and a square conducting pipe with a full width which is 3 times the semimajor axis of the beam.

Shown in Figs. 2(a) and 2(b), the dotted curves are the envelopes and angle of the beam ellipse obtained from the PFB2D simulation for the example of a fully relativistic elliptic beam with $V_{b}=198.5 \mathrm{keV}$, current $I_{b}=85.5 \mathrm{~A}$, aspect ratio $a / b=5$, and nonaxisymmetric periodic permanent magnet focusing with $B_{0}=2.4 \mathrm{kG}, S=2.2 \mathrm{~cm}$, and $k_{0 y} / k_{0 x}=1.52$. In the simulation, the emittance is set to be negligibly small in order to demonstrate the cold-fluid approximation. As shown in Fig. 2, there is excellent agreement between the theoretical envelope solution (solid curves) and the self-consistent PIC simulation results (dotted curves).

The PFB2D simulation also shows that the transverse beam distribution preserves the equilibrium profile as it propagates. In Fig. 3, 10000 particles (a sample of the $5 \times$ $10^{5}$ particles in the PFB2D simulation) are plotted in the $(x, y)$ plane and $(x, d y / d s)$ plane for five snapshots within one period: $s / S=9.0,9.25,9.5,9.75$, and 10.0 for the same beam shown in Fig. 2. The preservation of the beam distribution is further demonstrated in Fig. 4, which displays the evolution of the normalized fourth moments $\left\langle x^{4}\right\rangle /\left\langle x^{2}\right\rangle^{2}$ and $\left\langle y^{4}\right\rangle /\left\langle y^{2}\right\rangle^{2}$ for the same beam shown in Fig. 2. In Fig. 4 , it is evident that the normalized fourth moments $\left\langle x^{4}\right\rangle /\left\langle x^{2}\right\rangle^{2}$ and $\left\langle y^{4}\right\rangle /\left\langle y^{2}\right\rangle^{2}$, which are both equal to 2 for a uniform-density distribution, remain to be 2 within $\pm 1 \%$ as the beam propagates for ten periods. The PIC simulation results suggest that the beam equilibrium is stable.

\section{CONCLUSION}

In conclusion, a novel exact paraxial cold-fluid equilibrium was found for a high-intensity, space-charge-dominated charged-particle beam with a periodically twisted elliptic cross section in a nonaxisymmetric periodic magnetic field. Generalized envelope equations, which determine the beam envelopes, ellipse orientation, density, and internal flow velocity profiles, were derived, and solved numerically for nonrelativistic and relativistic examples of such beams. The equilibrium and stability of such beams were demonstrated by self-consistent particle-in-cell (PIC) simulations. We anticipate that the equilibrium theory will provide a valuable tool in the design of high-intensity elliptic beams in novel vacuum electronic devices, especially for ribbon-beam klystrons and traveling-wave tubes. 

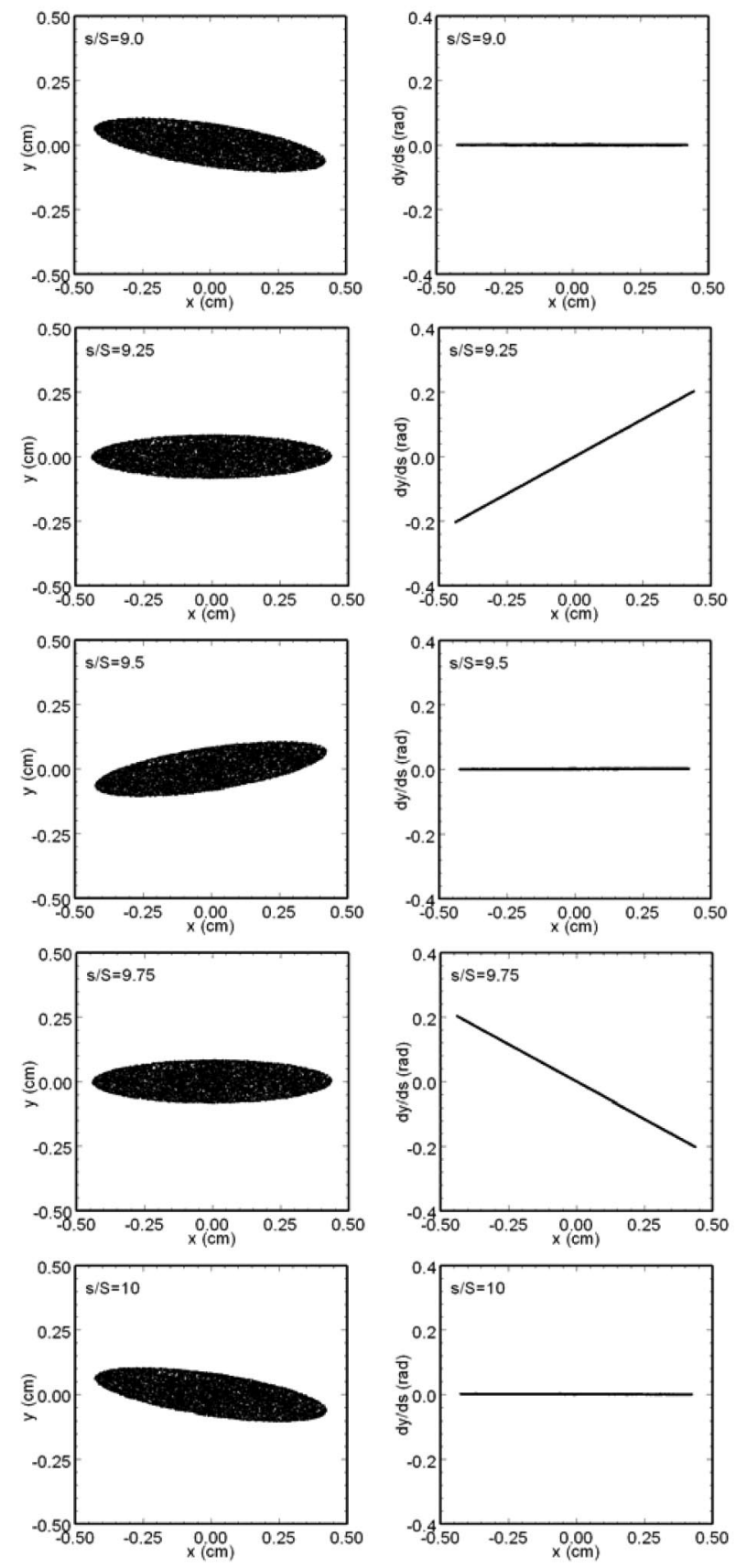

FIG. 3. Plots of 10000 particles (a sample of the $5 \times 10^{5}$ particles in the PFB2D simulation) in the $(x, y)$ plane and $(x, d y / d s)$ plane for five snapshots within one period: $s / S=$ 9.0, 9.25, 9.5, 9.75, and 10.0 for the same beam shown in Fig. 2.

The ellipse-shaped beam equilibrium may provide some flexibility in the design and operation of high-intensity accelerators.

\section{ACKNOWLEDGMENTS}

This work was supported by U.S. Department of Energy, Office of High-Energy Physics, Grant No. DE-FG0295ER40919, Office of Fusion Energy Science, Grant

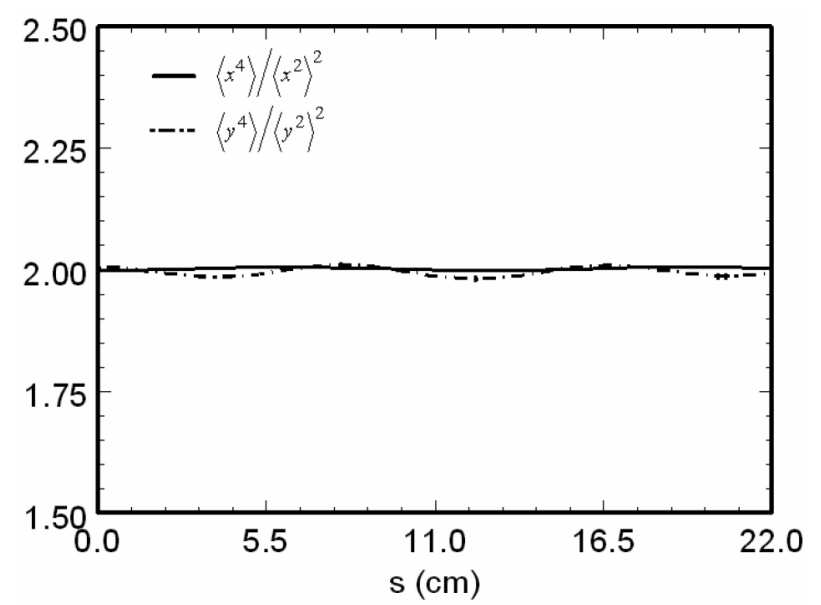

FIG. 4. Plots of the evolution of normalized fourth moments $\left\langle x^{4}\right\rangle /\left\langle x^{2}\right\rangle^{2}$ and $\left\langle y^{4}\right\rangle /\left\langle y^{2}\right\rangle^{2}$ for the same beam shown in Fig. 2.

No. DE-FG 02-01-ER54662, Air Force Office of Scientific Research, Grant No. F49620-03-1-0230, and the MIT Deshpande Center for Technological Innovation.

\section{APPENDIX}

Substituting Eqs. (5) and (6) into Eq. (1) and expressing the result in terms of the tilde coordinates, we obtain

$$
\begin{aligned}
& \left(\mu_{x}+\mu_{y}-\frac{a^{\prime}}{a}-\frac{b^{\prime}}{b}\right) \Theta\left(1-\frac{\tilde{x}^{2}}{a^{2}}-\frac{\tilde{y}^{2}}{b^{2}}\right) \\
& +2\left[\left(\frac{a^{\prime}}{a}-\mu_{x}\right) \frac{\tilde{x}^{2}}{a^{2}}+\left(\frac{b^{\prime}}{b}-\mu_{y}\right) \frac{\tilde{y}^{2}}{b^{2}}\right. \\
& \left.+\left(-\frac{b}{a} \theta^{\prime}+\frac{a}{b} \theta^{\prime}+\frac{b}{a} \alpha_{x}-\frac{a}{b} \alpha_{y}\right) \frac{\tilde{x}}{a} \frac{\tilde{y}}{b}\right] \\
& \times \delta\left(1-\frac{\tilde{x}^{2}}{a^{2}}-\frac{\tilde{y}^{2}}{b^{2}}\right)=0,
\end{aligned}
$$

where the prime denotes $\partial / \partial s$, and the relations $\tilde{x}^{\prime}=\tilde{y} \theta^{\prime}$, $\tilde{y}^{\prime}=-\tilde{x} \theta^{\prime}, \quad \partial \mathbf{e}_{\tilde{x}} / \partial s=\mathbf{e}_{\tilde{y}} \theta^{\prime}$ and $\partial \mathbf{e}_{\tilde{y}} / \partial s=-\mathbf{e}_{\tilde{x}} \theta^{\prime}$ have been used. Since Eq. (A1) must be satisfied for all $\tilde{x}$ and $\tilde{y}$, the coefficients of the terms proportional to $\Theta, \tilde{x}^{2} \delta, \tilde{y}^{2} \delta$ and $\tilde{x} \tilde{y} \delta$ must vanish independently. This leads to the following equations:

$$
\begin{gathered}
\mu_{x}=\frac{1}{a} \frac{d a}{d s}, \\
\mu_{y}=\frac{1}{b} \frac{d b}{d s}, \\
\frac{d \theta}{d s}=\frac{a^{2} \alpha_{y}-b^{2} \alpha_{y}}{a^{2}-b^{2}} .
\end{gathered}
$$

The force balance equation (3) represents two equations, one in the direction $\mathbf{e}_{\tilde{x}}$ and the other in the $\mathbf{e}_{\tilde{y}}$ direction. Substituting Eqs. (4)-(7) into Eq. (3), the two equations can be expressed as 


$$
\begin{aligned}
& f_{x} \tilde{x}+g_{y} \tilde{y}=0, \\
& g_{x} \tilde{x}+f_{y} \tilde{y}=0,
\end{aligned}
$$

where

$$
\begin{aligned}
f_{x}= & \mu_{x}^{2}+\mu_{x}^{\prime}-\alpha_{x} \alpha_{y}+\left(\alpha_{x}-\alpha_{y}\right) \theta^{\prime}-\frac{2 K}{(a+b)} \\
& -2 \sqrt{\kappa_{z 0}} \sin \left(k_{0} s\right) \alpha_{y} \\
+ & \sqrt{\kappa_{z 0}} \frac{k_{0 x}^{2}-k_{0 y}^{2}}{k_{0}} \sin (2 \theta) \cos \left(k_{0} s\right), \\
f_{y}= & \mu_{y}^{2}+\mu_{y}^{\prime}-\alpha_{x} \alpha_{y}-\left(\alpha_{x}-\alpha_{y}\right) \theta^{\prime}-\frac{2 K}{(a+b)} \\
+ & 2 \sqrt{\kappa_{z 0}} \sin \left(k_{0} s\right) \alpha_{x} \\
+ & \sqrt{\kappa_{z 0}} \frac{k_{0 x}^{2}-k_{0 y}^{2}}{k_{0}} \sin (2 \theta) \cos \left(k_{0} s\right), \\
g_{x}= & \alpha_{y}^{\prime}+\left(\mu_{x}-\mu_{y}\right) \theta^{\prime}+\left(\mu_{x}+\mu_{y}\right) \alpha_{y} \\
& -2 \sqrt{\kappa_{z 0}}\left(\frac{k_{0 x}^{2}}{k_{0}} \cos ^{2} \theta+\frac{k_{0 y}^{2}}{k_{0}} \sin ^{2} \theta\right) \cos \left(k_{0} s\right) \\
& -2 \sqrt{\kappa_{z 0}} \mu_{x} \sin \left(k_{0} s\right), \\
g_{y}= & -\alpha_{x}^{\prime}+\left(\mu_{x}-\mu_{y}\right) \theta^{\prime}-\left(\mu_{x}+\mu_{y}\right) \alpha_{x} \\
& +2 \sqrt{\kappa_{z 0}}\left(\frac{k_{0 x}^{2}}{k_{0}} \sin ^{2} \theta+\frac{k_{0 y}^{2}}{k_{0}} \cos ^{2} \theta\right) \cos \left(k_{0} s\right) \\
& +2 \sqrt{\kappa_{z 0}} \mu_{y} \sin \left(k_{0} s\right) .
\end{aligned}
$$

Since Eqs. (A5) and (A6) must be satisfied for all $\tilde{x}$ and $\tilde{y}$, the coefficients of the terms proportional to $\tilde{x}$ and $\tilde{y}$ must vanish independently, which lead to

$$
\begin{aligned}
& f_{x}=0, \\
& f_{y}=0,
\end{aligned}
$$

$$
\begin{aligned}
& g_{x}=0, \\
& g_{y}=0 .
\end{aligned}
$$

After substituting Eqs. (A2) and (A3) into Eqs. (A11)(A14), we obtain the generalized envelope equations (A8)(A12).

[1] P. A. Sturrock, J. Electron. Control 7, 162 (1959).

[2] Z.X. Zhang, V. L. Granatstein, W. W. Destler et al., IEEE Trans. Plasma Sci. 21, 760 (1993).

[3] J.H. Booske, B.D. McVey, and T.M. Antonsen, Jr., J. Appl. Phys. 73, 4140 (1993).

[4] J.H. Booske, A. H. Kumbasar, and M. A. Basten, Phys. Rev. Lett. 71, 3979 (1993).

[5] J.H. Booske and M. A. Basten, IEEE Trans. Plasma Sci. 27, 134 (1999).

[6] M. A. Basten and J.H. Booske, J. Appl. Phys. 85, 6313 (1999).

[7] F. J. Sacherer, Ph.D. thesis, Lawrence Radiation Laboratory, University of California, Berkeley, 1968, pp. 15-19.

[8] V. Danilov, S. Cousineau, S. Henderson, and J. Holmes, Phys. Rev. ST Accel. Beams 6, 094202 (2003).

[9] I. M. Kapchinskij, and V. V. Vladimirskij, in Proceedings of the International Conference on High Energy Accelerators (CERN, Geneva, 1959), p. 274.

[10] R. C. Davidson, Physics of Nonneutral Plasma (AddisonWesley, Reading, MA, 1990), p. 694.

[11] C. Chen, R. Pakter, and R. C. Davidson, Phys. Rev. Lett. 79, 225 (1997).

[12] R.C. Davidson and H. Qin, Physics of Intense Charged Particle Beams in High Energy Accelerators (World Scientific, Singapore, 2001), p. 242.

[13] R. Pakter and C. Chen, Phys. Rev. E 62, 2789 (2000).

[14] C. Chen and R. Pakter, Phys. Plasmas 7, 2203 (2000).

[15] R. C. Davidson, P. Stoltz, and C. Chen, Phys. Plasmas 4, 3710 (1997).

[16] R. Bhatt and C. Chen, Phys. Rev. ST Accel. Beams 8, 014201 (2005). 